\title{
PIN Diode and Neutron Spectrum Measurements at the Army Pulse Radiation Facility
}

\author{
M.A. Oliver \\ Aberdeen Proving Ground, MD
}

\begin{abstract}
Neutron spectrum measurements using the foil activation technique have been made in two widely varying environments. One is an extremely high neutron-to-gamma field and the other extremely low. These measurements were used to characterize the fields and to evaluate the use of the DN-156 PIN diode for measuring $1 \mathrm{MeV}$ equivalent neutron fluence in silicon $(\Phi 1 \mathrm{MeV}(\mathrm{Si}))$. The agreement between the $\Phi 1 \mathrm{MeV}(\mathrm{Si})$ as measured with diodes and as determined by the spectral measurements was within $\pm 5 \%$. A proton recoil neutron spectrum measurement was also made in the low gamma environment.
\end{abstract}

\section{INTRODUCTION}

The Army Pulse Radiation Facility (APRF) is a multi-faceted facility operating a Godiva type pulse reactor, housed in a low scatter aluminum silo $30 \mathrm{~m}$ in diameter and $20 \mathrm{~m}$ high. The reactor is moveable to several locations and heights. Several well characterized exposure environments are available for experiments.

When testing silicon devices against a nuclear threat, it is essential to consider the difference between the neutron energy spectrum of the threat environment and that of the test environment. This is accounted for by defining a quantity that would have the same damage effect as if all the incident neutrons were $1 \mathrm{MeV}$ in energy. This quantity is defined as the $1 \mathrm{MeV}$ equivalent neutron fluence in silicon $(\Phi 1 \mathrm{MeV}(\mathrm{Si})$, Equation 1), where $\Phi(E)$ is the incident neutron spectrum, $K_{D}$ is the silicon neutron damage function and $\overline{\mathrm{K}}_{\mathrm{D}}(1 \mathrm{MeV})$ is the ASTM reference silicon damage at $1 \mathrm{MeV}$ [1].

$$
\Phi(1 \mathrm{MeV})=\frac{\int_{0}^{\infty} \Phi(E) K_{D}(E) d E}{\bar{K}_{D}(1 \mathrm{MeV})}
$$

Currently, silicon DN-156 PIN diodes are being used to measure $\Phi 1 \mathrm{MeV}(\mathrm{Si})$ directly $[2,3]$. The purpose of this work is to characterize two neutron environments and to evaluate this technique by comparing it to the results of a foil activation spectrum measurement.

Two neutron spectral measurements were made using the foil activation technique. Due to the size of the supporting electronic equipment and the limited volume of the device under test (DUT), foil exposures were not made inside the DUT. DN-156 PIN diodes [2,3] and small sulfur pellets were exposed in the DUT to monitor $\Phi 1 \mathrm{MeV}(\mathrm{Si})$ during the test. The foil measurements were made in mock-ups of the original item, which were also monitored with DN-156 PIN diodes and sulfur pellets.

\section{TEST CONFIGURATIONS}

\section{A. $12.7 \mathrm{~cm}$ Cd-Poly Converter}

The first environment was with the DUT directly behind a $12.7 \mathrm{~cm}$ thick gamma converter made of cadmiumloaded ( $5 \%$ by weight) polyethylene. The converter is $46 \mathrm{~cm}$ square. The reactor was placed $2.54 \mathrm{~cm}$ in front of the face of the converter and centered on the DUT.

Neutrons are moderated by the polyethylene and interact with the cadmium, producing capture gamma rays. This produces a field with a very low neutron-to-gamma ratio.

Lead and polyethylene shielding materials were used behind the test setup to simulate the shielding used in the actual test.

\section{B. $10.2 \mathrm{~cm}$ Bismuth shield}

In order to create an environment suitable for both activation foils and a proton recoil spectrometer, ROSPEC [4] the mock-up geometry was different from the actual test. A $10.2 \mathrm{~cm}$ wall of bismuth bricks was constructed and the detectors placed $50 \mathrm{~cm}$ behind it. In the actual test $10.2 \mathrm{~cm}$ of bismuth was used but the DUT was directly behind it. Both the foils and the DN-156 PIN diodes were irradiated at the same location.

Bismuth has a very low neutron scatter cross section making it very efficient in transmitting neutrons. It is high $\mathrm{Z}$ material (83) making it a very good gamma ray shield. The combination produces a very high neutron-to-gamma field.

\section{FOIL MEASUREMENTS}

\section{A. Exposures}

Twelve to 14 foils were exposed in each environment, covering the full range of neutron energies. Table I shows these reactions and the $5 \%$ to $95 \%$ energy limits of the foil response. Reactions marked with an * were used in the 
indicated configurations. The foils were exposed in sets of 4 or less which required multiple reactor operations. These operations were monitored with sulfur pellets located in two reference positions. DN-156 diodes and sulfurs were also exposed with the foils. The diodes and sulfurs are independent monitors being compared to the results of the foil measurements and are therefore not used as foil sensors.

\section{B. Foil Counting}

Gamma lines from all the foils were counted on a high purity germanium detector (HPGe). The detector was calibrated in several fixed, reproducible geometries using a mixed nuclide source traceable to NIST. Each foil was counted twice at distances far enough from the detector to insure that detector dead time and summing corrections were $<1 \%$.

Foil activities were determined as follows [6]:

$$
A_{0}=\frac{C \lambda^{2} t_{i} e^{\lambda t_{W M}}}{\epsilon P_{Y} F_{y} A_{i}\left(1-e^{-\lambda t_{i}}\right)\left(1-e^{-\lambda t_{c}}\right) N m}
$$

where:

$A_{o}=\mathrm{dps} /$ atom or fissions/atom

$\mathrm{C}=$ counts under photo peak

$\lambda=$ decay constant of product nuclei $\left(\mathrm{s}^{-1}\right)$

$t_{i}=$ time of irradiation (s)

$t_{w}=$ time from end of irradiation to start of count (s)

$t_{c}=$ time of count (s)

$\mathbf{M}=$ atomic mass of target nuclei

$\epsilon=$ detector efficiency

$\mathrm{P}_{\gamma}=$ fraction of $\gamma$ emission during decay

$F_{y}=$ fission yield ( 1 for activation foils)

$A_{i}=$ isotopic abundance

$\mathrm{N}=$ Avogadro's number

$\mathrm{m}=$ mass of foil $(\mathrm{g})$

Fission foil decay correction is complicated by parent daughter decay scheme if ${ }^{140} \mathrm{La}$ line is counted [7].

The resulting activities were used as input into the SANDII spectrum adjustment code [8].

\section{SANDII Analysis}

SANDII requires 3 inputs; a set of reaction cross sections, a corresponding set of foil activities and an initial input spectrum. The code calculates activities based on these data and compares the calculated and measured activities. If the standard deviation between measured and calculated activities for all the reactions is not within acceptable (user determined) limits the code adjusts the initial spectrum in the areas of poor agreement and makes a new comparison of activities. It continues to iterate in this manner until the preset standard deviation is achieved.

In this work the ENDF/B-V cross section set was used and initial input spectrum from the SPRIII and ACRR [7] reactors at Sandia National Laboratories were used. These input spectra were used because they represented very similar neutron environments to the ones being measured. Better than $4 \%$ standard deviation was achieved with 2 and 7 iterations for the $\mathrm{Cd}$-poly and $\mathrm{Bi}$ fields respectively.

\section{DIODE \& SULFUR MEASUREMENTS}

\section{A. Diodes}

The DN-156 PIN diode has several advantages. It is small, fitting inside electronic sub-assemblies easily. It is easy to use. Most importantly it measures $\Phi 1 \mathrm{MeV}(\mathrm{Si})$ directly when properly calibrated. It has been shown to have little or no sensitivity to thermal neutrons or gamma rays $[2,3]$. The main disadvantage is that they saturate at about $10^{12} \mathrm{n} / \mathrm{cm}^{2}$. Although work is being done to extend this range [3], this usually requires a separate low level run to characterize an environment.

APRF diodes are calibrated in a NIST ${ }^{252} \mathrm{Cf}$ field in terms of $\Phi 1 \mathrm{MeV}$ to $\pm 9 \%$. They were temperature controlled $\left(20^{\circ} \mathrm{C}\right)$ and read out 24 hours after exposure so no temperature or fading corrections were needed.

\section{B. Sulfur Pellets}

Sulfur pellets are primarily sensitive to neutrons with energies $>3 \mathrm{MeV}$. When sulfur results are used to report the spectrum 3-MeV fluence $(\Phi(>3 \mathrm{MeV}))$ in a spectrum that differs greatly from a ${ }^{252} \mathrm{Cf}$ spectrum (the calibration spectrum) they are corrected for this spectral difference using the ratio of spectrum averaged cross section $(\bar{\sigma})$ of $\operatorname{sulfur}(>3 \mathrm{MeV})$ in ${ }^{252} \mathrm{Cf}$ to that in the test spectrum [9]. A $3-\mathrm{MeV} \bar{\sigma}$ for the ${ }^{32} \mathrm{~S}(\mathrm{n}, \mathrm{p}){ }^{32} \mathrm{P}$ reaction can be defined as:

$$
\bar{\sigma}[3 \mathrm{MeV}]=\frac{\int^{\infty} \Phi(E) \sigma_{32 S}(E) d E}{\int_{3 \mathrm{MeV}}^{\infty} \Phi(E) d E}
$$

where $\sigma_{32 s}(E)$ is the $\left.{ }^{32} S(n, p)\right)^{32} P$ cross section and $\Phi(E)$ is the neutron spectrum. The denominator of Equation 3 is referred to as the spectrum $3-\mathrm{MeV}$ fluence. The $\bar{\sigma}(\mathrm{Cf})$, calculated from the NIST ${ }^{252} \mathrm{Cf}$ spectrum [10] and the IRDF-90 sulfur cross section [11] is $299 \mathrm{mb}$.

The APRF sulfur system is calibrated in a NIST ${ }^{252} \mathrm{Cf}$ field in terms of $\Phi(>3 \mathrm{MeV})_{\mathrm{Cf}}$ to $\pm 3 \%$. The corrected $\Phi(>3 \mathrm{MeV})$ is accurate to $\pm 10 \%$.

\section{C. $1 \mathrm{MeV}$ to Sulfur Ratio}

In radiation-hardness testing of electronics, neutron fluence is reported in terms of $1 \mathrm{MeV}$ (Si) equivalent fluence 


\begin{tabular}{|c|c|c|c|c|c|}
\hline \multicolumn{6}{|c|}{ TABLE I. REACTIONS USED IN SANDII ANALYSIS } \\
$\begin{array}{c}\text { FEIL } \\
\text { RECTION }\end{array}$ & COVERS & $\begin{array}{c}\text { LOW } \\
\text { ENERGY } \\
(\mathrm{MeV})\end{array}$ & $\begin{array}{c}\text { HIGH } \\
\text { ENERGY } \\
(\mathrm{MeV})\end{array}$ & $\begin{array}{c}5^{\prime \prime} \mathrm{Cd} \\
\text { Poly }\end{array}$ & $4^{*} \mathrm{Bi}$ \\
\hline${ }^{23} \mathrm{Na}(\mathrm{n}, \gamma)^{24} \mathrm{Na}$ & $\mathrm{None}$ & $1.5 \times 10^{-5}$ & $2.1 \times 10^{-1}$ & $*$ & \\
\hline${ }^{23} \mathrm{Na}(\mathrm{n}, \gamma)^{24} \mathrm{Na}$ & $\mathrm{Cd}$ & $1.5 \times 10^{-5}$ & $2.1 \times 10^{-1}$ & $*$ & $*$ \\
\hline${ }^{24} \mathrm{Mg}(\mathrm{n}, \mathrm{p})^{24} \mathrm{Na}$ & $\mathrm{Cd}$ & 6.5 & 11.7 & $*$ & $*$ \\
\hline${ }^{27} \mathrm{Al}(\mathrm{n}, \alpha)^{24} \mathrm{Na}$ & $\mathrm{Cd}$ & 6.4 & 12.1 & $*$ & $*$ \\
\hline${ }^{46} \mathrm{Ti}(\mathrm{n}, \mathrm{p})^{46} \mathrm{Sc}$ & $\mathrm{Cd}$ & 3.7 & 9.1 & $*$ & $*$ \\
\hline${ }^{47} \mathrm{Ti}(\mathrm{n}, \mathrm{p})^{47} \mathrm{Sc}$ & $\mathrm{Cd}$ & 1.7 & 6.8 & $*$ & $*$ \\
\hline${ }^{48} \mathrm{Ti}(\mathrm{n}, \mathrm{p})^{48} \mathrm{Sc}$ & $\mathrm{Cd}$ & 5.8 & 12.3 & $*$ & $*$ \\
\hline${ }^{54} \mathrm{Fe}(\mathrm{n}, \mathrm{p})^{54} \mathrm{Mn}$ & $\mathrm{Cd}$ & 2.1 & 7.1 & & $*$ \\
\hline${ }^{56} \mathrm{Fe}(\mathrm{n}, \mathrm{p})^{56} \mathrm{Mn}$ & $\mathrm{Cd}$ & 5.4 & 11.2 & & $*$ \\
\hline${ }^{58} \mathrm{Ni}(\mathrm{n}, \mathrm{p})^{58} \mathrm{Co}$ & $\mathrm{Cd}$ & 1.8 & 7.0 & $*$ & $*$ \\
\hline${ }^{197} \mathrm{Au}(\mathrm{n}, \gamma)^{198} \mathrm{Au}$ & $\mathrm{Cd}$ & $4.5 \times 10^{-6}$ & $1.2 \times 10^{-2}$ & $*$ & $*$ \\
\hline${ }^{197} \mathrm{Au}(\mathrm{n}, \gamma)^{198} \mathrm{Au}$ & $\mathrm{None}$ & $4.5 \times 10^{-6}$ & $1.2 \times 10^{-2}$ & $*$ & $*$ \\
\hline${ }^{237} \mathrm{~Np}(\mathrm{n}, \mathrm{f})^{140} \mathrm{La}$ & $\mathrm{Cd} \&{ }^{10} \mathrm{~B}$ & $5.0 \times 10^{-1}$ & 4.5 & $*$ & $*$ \\
\hline${ }^{238} \mathrm{U}(\mathrm{n}, \mathrm{f})^{140} \mathrm{La}$ & $\mathrm{Cd} \&{ }^{10} \mathrm{~B}$ & 1.4 & 5.9 & & $*$ \\
\hline${ }^{239} \mathrm{Pu}(\mathrm{n}, \mathrm{f})^{140} \mathrm{La}$ & $\mathrm{Cd} \mathrm{\&}{ }^{10} \mathrm{~B}$ & $1.4 \times 10^{-2}$ & 3.4 & $*$ & $*$ \\
\hline
\end{tabular}

[1]. Neutron exposures are monitored using sulfur pellets. In order to convert fluence as determined by sulfur activation to $\Phi 1 \mathrm{MeV}(\mathrm{Si})$, the ratio of $\Phi 1 \mathrm{MeV}(\mathrm{Si})$ to $\Phi$ (sulfur) must be determined in the field that the monitor was exposed in.

There are two ways to determine this ratio. One is to determine the neutron energy spectrum in the test field and take the ratio of $\Phi 1 \mathrm{MeV}$ to $\Phi(>3 \mathrm{MeV})$. The other is to expose a sulfur pellet along with a calibrated silicon device $[2,3,5]$ in the test field. The APRF uses these monitors to measure the $1 \mathrm{MeV} / \mathrm{Sulfur}$ ratio directly (Equation 4 .)

$$
1 \mathrm{MeV} / \mathrm{Sulfur}=\frac{\Phi 1 \mathrm{MeV} V_{\text {diode }}}{\Phi(>3 \mathrm{MeV})_{\text {sulfur }}}
$$

When sulfur pellets are used with the $1 \mathrm{MeV} /$ Sulfur ratio to report $\Phi 1 \mathrm{MeV}(\mathrm{Si})$ no corrections for differences in $\bar{\sigma}$ are needed. This is because the $\Phi(>3 \mathrm{MeV})$ is not determined in an absolute sense but only to relate two reactor irradiations.

The $1 \mathrm{MeV} / \mathrm{Sulfur}$ ratio is established once. As long as the exposure geometry and neutron environment, as well as the procedure for determining the sulfur activation, remain constant, this ratio can be used in subsequent irradiations to determine $\Phi 1 \mathrm{MeV}(\mathrm{Si})$ from the activity of a sulfur monitor.
The stability of the sulfur counting system is $\pm 2 \%$ while the diode calibration is $\pm 9 \%$ making the uncertainty of the $1 \mathrm{MeV} /$ Sulfur ratio $\pm 10 \%$

\section{ROSPEC MEASUREMENTS}

ROSPEC [4] is a self-contained proton recoil neutron spectrometer. Four spherical proportional counters are optimized to cover an energy range from $0.1 \mathrm{MeV}$ to 4.5 $\mathrm{MeV}$. Each detector was irradiated separately $50 \mathrm{~cm}$ behind $10.7 \mathrm{~cm}$ of bismuth.

Dead time problems prevented measurements in high background fields close to the reactor. The reactor was run at the lowest reproducible levels $(0.1$ watt) and results were normalized by reactor power.

\section{RESULTS}

\section{A. Spectrum Characteristics}

All data reported here has been normalized to a typical reactor pulse of $1.2 \times 10^{17}$ fissions, which is comparable to $57 \mathrm{kWmin}$ of steady state operation. Several integral parameters are of interest in neutron environments. Table II lists these results. It should be noted that both the 
Table II. Spectral Characteristics.

\begin{tabular}{||l|l|l|l||}
\hline & APRF-FF & CD-POLY & BISMUTH \\
\hline$\Phi$ Total $\left(\mathrm{n} / \mathrm{cm}^{2}\right)$ & $2.77 \times 10^{12}$ & $2.29 \times 10^{12}$ & $1.64 \times 10^{12}$ \\
\hline$\Phi(>10 \mathrm{keV})$ & $2.75 \times 10^{12}$ & $1.53 \times 10^{12}$ & $1.57 \times 10^{12}$ \\
\hline$\Phi(>3 \mathrm{MeV})$ & $3.53 \times 10^{11}$ & $2.41 \times 10^{11}$ & $1.09 \times 10^{11}$ \\
\hline$\Phi 1 \mathrm{MeV}(\mathrm{Si}-93)$ & $2.50 \times 10^{12}$ & $1.22 \times 10^{12}$ & $1.30 \times 10^{12}$ \\
\hline$\Phi 1 \mathrm{MeV}(\mathrm{GaAs})$ & $2.58 \times 10^{12}$ & $1.30 \times 10^{12}$ & $1.37 \times 10^{12}$ \\
\hline$\Phi 1 \mathrm{MeV}(\mathrm{Si}-85)$ & $2.67 \times 10^{12}$ & $1.32 \times 10^{12}$ & $1.35 \times 10^{12}$ \\
\hline$\Phi 1 \mathrm{MeV} / \Phi(>3 \mathrm{MeV})$ & 7.08 & 5.06 & 11.93 \\
\hline $\mathrm{HP}(\mathrm{Si}-93)$ & 0.91 & 0.80 & 0.83 \\
\hline $\bar{\sigma} \mathrm{Sulfur}>3 \mathrm{McV}(\mathrm{b})$ & 0.303 & 0.296 & 0.324 \\
\hline$\Phi 1 \mathrm{MeV}(\mathrm{Si}-93) / \mathrm{cGy}(\mathrm{Si}, \gamma)$ & $3.61 \times 10^{9}$ & $1.02 \times 10^{8}$ & $1.67 \times 10^{10}$ \\
\hline
\end{tabular}

Figure 1. Full Spectrum comparison.

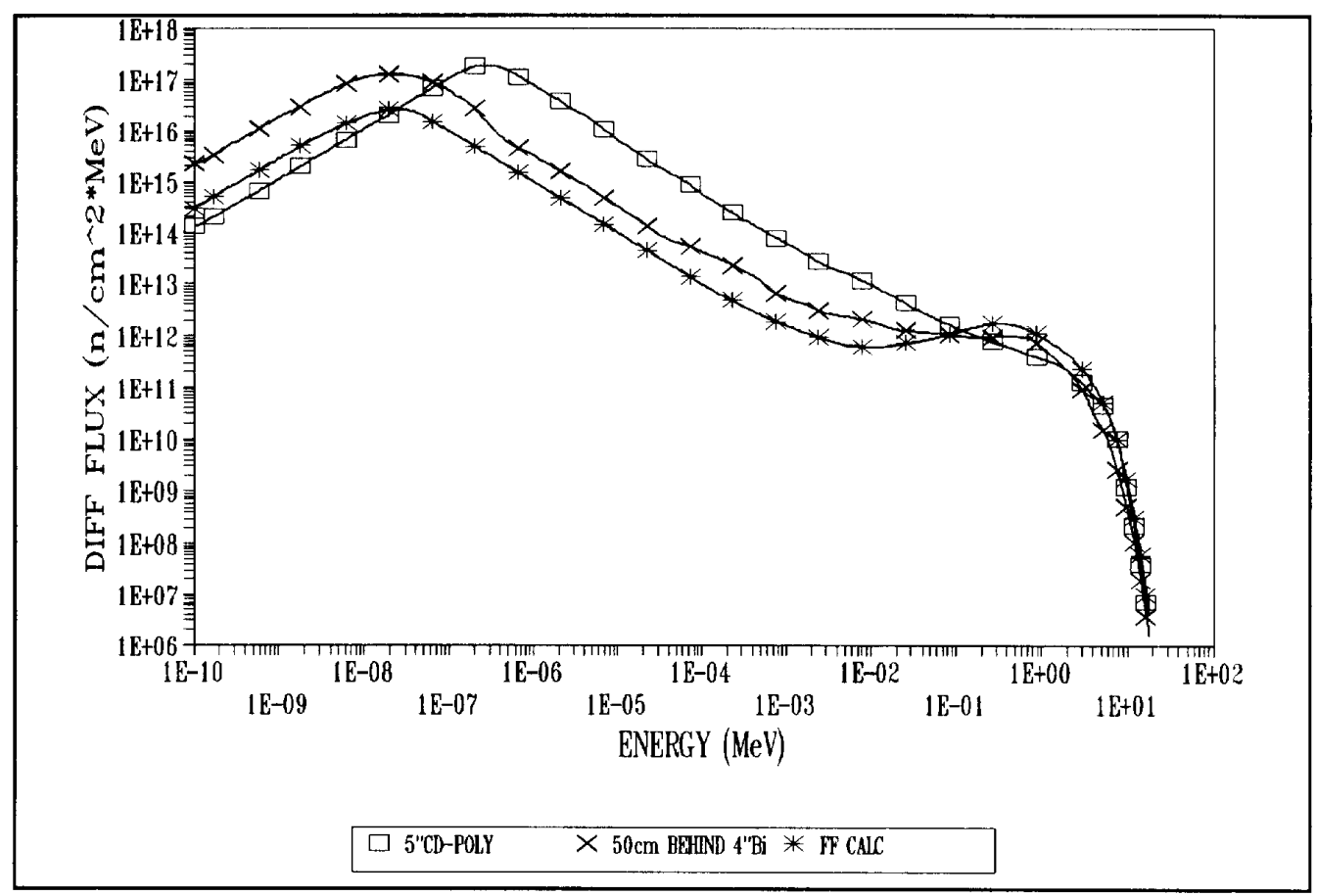


Figure 2. Fast neutron spectrum comparison.

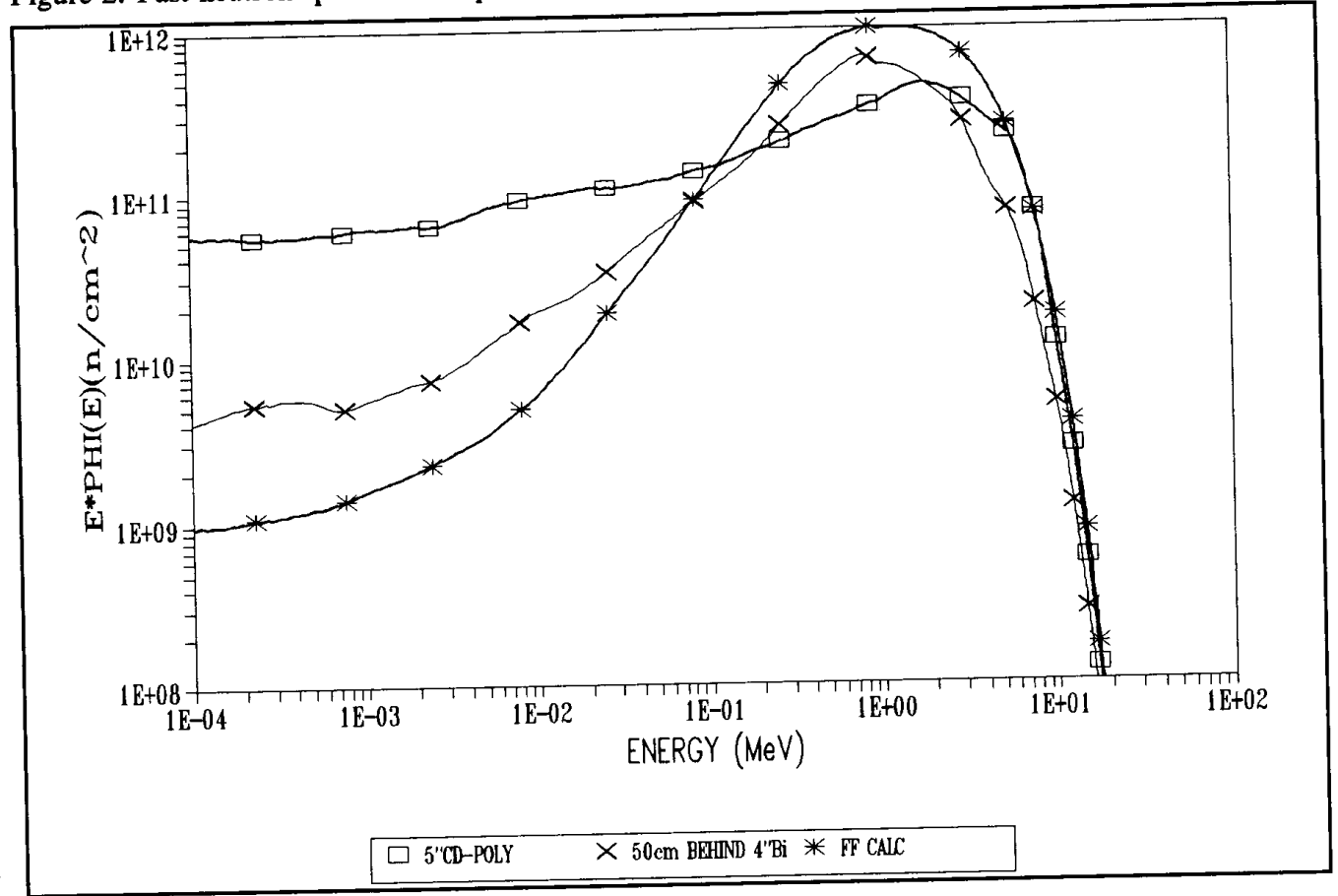

TABLE III. DIODE, SULFUR \& FOIL AGREEMENT

\begin{tabular}{|c|c|c|c|c|}
\hline & & FOIL RESULTS & $\begin{array}{l}\text { DIODE \& } \\
\text { SULFUR }\end{array}$ & $\begin{array}{c}\% \\
\text { DIFF }\end{array}$ \\
\hline \multirow[t]{3}{*}{ 4" BISMUTH } & $\Phi 1 \mathrm{MeV}(\mathrm{Si}-93) \mathrm{n} / \mathrm{cm}^{2}$ & $1.30 \times 10^{12}$ & $1.28 \times 10^{12}$ & 1.6 \\
\hline & $\begin{array}{l}\Phi(>3 \mathrm{MeV}) \mathrm{n} / \mathrm{cm}^{2} \\
\text { (Cf equivalent) }\end{array}$ & $1.18 \times 10^{11}$ & $1.13 \times 10^{11}$ & 4.4 \\
\hline & $1 \mathrm{MeV} /$ Sulfur & 11.0 & 12.3 & -3.5 \\
\hline \multirow[t]{3}{*}{ 5" Cd-Poly } & $\Phi 1 \mathrm{MeV}(\mathrm{Si}-93) \mathrm{n} / \mathrm{cm}^{2}$ & $1.22 \times 10^{12}$ & $1.22 \times 10^{12}$ & -0.5 \\
\hline & $\begin{array}{l}\Phi(>3 \mathrm{MeV}) \mathrm{n} / \mathrm{cm}^{2} \\
\text { (Cf equivalent) }\end{array}$ & $2.39 \times 10^{11}$ & $2.37 \times 10^{11}$ & 0.1 \\
\hline & $1 \mathrm{MeV} /$ Sulfur & 5.1 & 5.2 & -1.9 \\
\hline
\end{tabular}


Figure 3. ROSPEC and foil spectra.

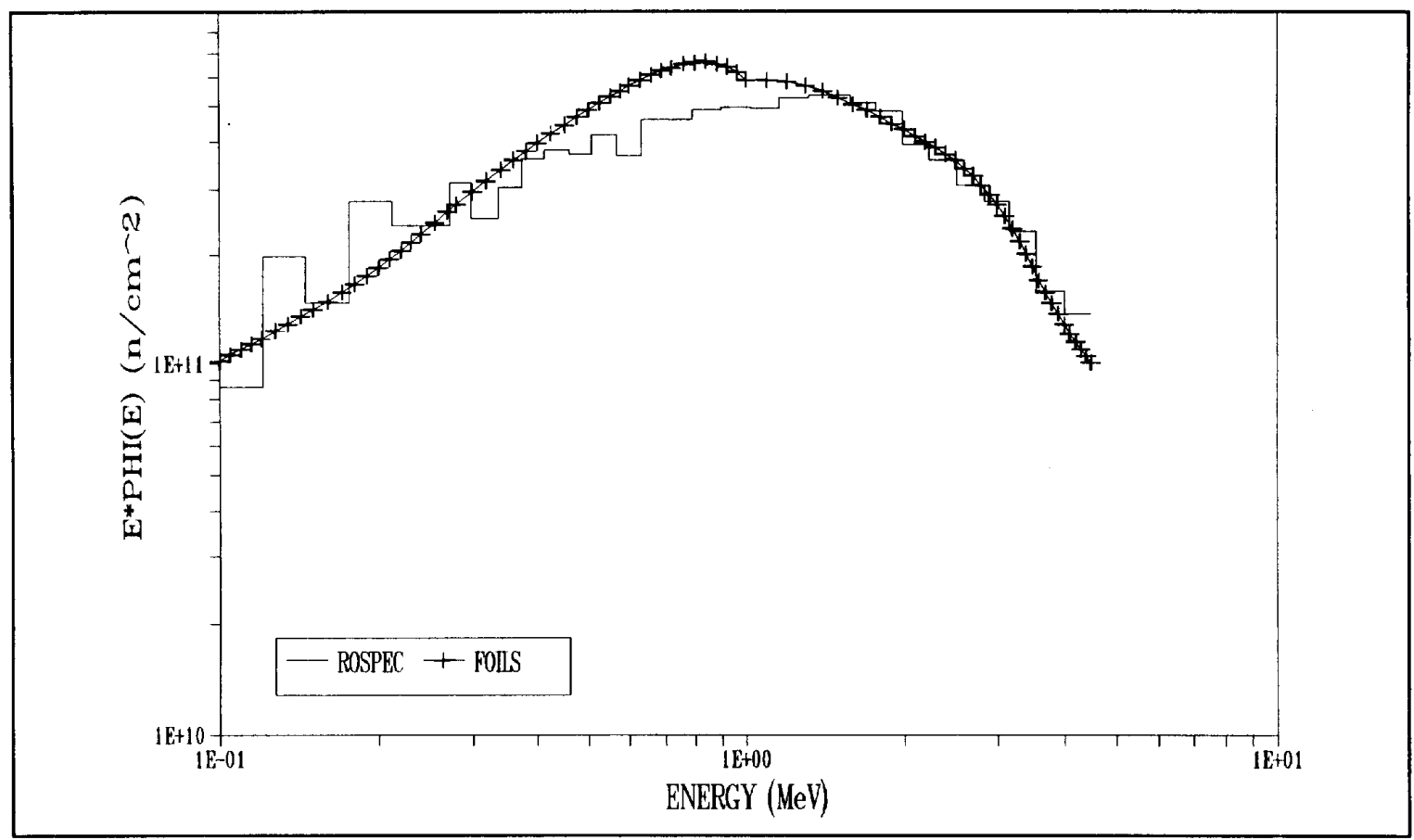

old, $1 \mathrm{MeV}(85)$, and the new, $1 \mathrm{MeV}(93)$ silicon damage functions are reported here. This is for comparison only, the new damage function is used in reporting $\Phi 1 \mathrm{MeV}$ (Si) [1]. Other useful quantities are the hardness parameter and neutron-to-gamma ratio. The first is the ratio of $\$ 1 \mathrm{MeV}(\mathrm{Si})$ / $\Phi(>10 \mathrm{keV})$ which is an indicator of the spectrum shape. The neutron-to-gamma ratio, expressed in terms of $\mathrm{n}^{\cdot \mathrm{cm}^{-2}}$ $/ \mathrm{cGy}(\mathrm{Si}, \gamma)$, is useful to know if the DUT maybe sensitive to $\gamma \mathrm{s}$.

Figures $1 \& 2$ show the resulting neutron spectra for the fields measured along with a free field calculation [12] for comparison. The full energy spectrum shows a substantial low energy "tail" from scattered neutrons. This is well below the threshold for silicon and has little or no impact on $\Phi 1 \mathrm{MeV}$. A more useful representation is given in Figure 2, of the energy range from $.01 \mathrm{MeV}$ to $20 \mathrm{MeV}$. Here the differences in spectrum "hardness" can be seen more clearly.

\section{B. Sulfur \& Diode}

The quantities measured with the sulfur and diode monitors show excellent agreement with full spectrum determinations above. Table III demonstrates this.

The critical parameter is the $1 \mathrm{MeV} / \mathrm{Sulfur}$ ratio which is used to report $\Phi 1 \mathrm{MeV}$ (Si) when only a sulfur pellet is used to monitor an exposure. These results are well within the $\pm 10 \%$ uncertainty assigned to $\Phi 1 \mathrm{MeV}(\mathrm{Si})$.

The quantity $\Phi(>3 \mathrm{MeV})$ has been corrected to compare ${ }^{252} \mathrm{Cf}$ equivalent fluence [9], this resulted in an $8 \%$ increase in the $\Phi(>3 \mathrm{MeV})$ reported by the foils in the bismuth environment and a $1 \%$ decrease in the Cd-poly environment.

\section{ROSPEC Results}

Due to the limited energy range, it was necessary to compare ROSPEC with the foil data from $0.1 \mathrm{MeV}$ to 4.5 $\mathrm{MeV}$. The integral parameter of interest is $\Phi 1 \mathrm{MeV}(\mathrm{Si})$,

$$
\frac{\int_{1 M e V}^{4.5 M e V} \Phi(E) K_{D}(E) d E}{\overline{K_{D}}(1 \mathrm{MeV})}
$$

Where $\Phi(E)$ is the incident neutron spectrum, $K_{D}(E)$ is the current $\mathrm{Si}$ damage cross section and $\overline{\mathrm{K}}_{\mathrm{D}}(1 \mathrm{MeV})$ is the ASTM defined reference damage [1]. This differs from the standard definition of $\Phi 1 \mathrm{MeV}$ only by the limits of integration.

Agreement was within 6\%, with ROSPEC reporting $1.21 \times 10^{12} \mathrm{n} / \mathrm{cm}^{2}$ and foils $1.28 \times 10^{12} \mathrm{n} / \mathrm{cm}^{2}$. Figure 3 shows a spectrum comparison of the ROSPEC spectrum with the foil 
spectrum over the energy range of interest. The discontinuity in the foil spectrum near $1 \mathrm{MeV}$ is not understood at this time. It is assumed to be an artifact of the computer code.

\section{CONCLUSIONS}

DN-156 PIN diodes may be used confidently to characterize a variety of neutron fields in terms of $1 \mathrm{MeV}(\mathrm{Si})$ equivalent neutron fluence. This becomes very significant when the DUT is a complex sub-assembly which cannot be subjected to the fluence required for activation foil exposures, or where the exposure volume limits the use of boron covered fission foils.

The APRF has acquired the capability of making neutron spectrum measurements using the activation foil technique. Two important neutron environments have been well characterized for use in radiation-hardness testing. These environments are not limited to electronic testing.

Proton recoil spectroscopy and foil activation techniques have been used successfully in the same radiation environment, providing confirmation of the foil measurements over a critical energy range.

\section{ACKNOWLEDGEMENTS}

The author would like to thank Wesley Sallee of White Sands Missile Range, and Jake Kelly of Sandia National Laboratory for their help in the implementation and use of the SANDII computer code. I would also like to thank Michael Stanka of the APRF for his expertise in making ROSPEC measurements in a very difficult environment.

\section{REFERENCES}

[1] ASTM Standard E722-93, "Standard Practice for Characterizing the Neutron Energy Fluence Spectra in Terms of an Equivalent Monoenergentic Neutron Fluence for Radiation-Hardness testing of Electronics, " 1993 Annual Book of ASTM Standards, Vol. 12.02, Oct 1993

[2] Heimbach, C.R., "The Use of Neutron-Sensitive Diodes as Monitors for 1-MeV Equivalent Neutrons, " Proceedings of the Seventh ASTMEURATOM Symposium on Reactor Dosimetry, Kluwer Academic Publishers, 1990

[3] Morin J. et al. , "Measuring $1 \mathrm{MeV}(\mathrm{Si})$ Equivalent Neutron Fluences with PIN Silicon Diodes," Proceeding of Second European Conference on Radiation and its Effects on Components and Systems, Saint-Malo, France 1993

[4] Stanka, M.B., "Spectroscopy 400 Meters from a Fission Neutron Source," from The Eighth ASTMEURATOM Symposium on Reactor Dosimetry, Vail, CO USA, 1993
[5] Kelly J.G. et al., "Use of Silicon Bipolar Transistors as Sensors for Neutron Energy Spectra Determinations," IEEE Trans. Nucl. Sci., Vol. NS-34,No.6, Dec. 1987.

ASTM Standard E721-93, "Standard Method for Determining Neutron Energy Spectra With NeutronActivation Foils for Radiation-Hardness testing of Electronics," 1993 Annual Book of ASTM Standards, Vol. 12.02, Oct 1993

[7] Kelly, J.G. \& Vehar, D.W., "Measurements of Neutron Spectra in Varied Environments by the Foil Activation Method with Arbitrary Trials, "SAND871330 , Sandia National Laboratories, Albuquerque, NM, Dec. 1987

[8] McElroy, W.N., et al., "A Computer-Automated Iterative Method for Neutron Flux Spectra Determination by Foil Activation," AFWL-TR-6741, Vol 1, Air Force Weapons Laboratory, Kirtland AFB, New Mexico, Sept. 1967.

[9] ASTM Standard E265-93, "Standard Test Method for Measuring Reaction Rates and Fast-Neutron Fluences by Radioactivation of Sulfur-32," 1993 Annual Book of ASTM Standards, Vol. 12.02, Oct 1993

[10] GrundI, J. \& Eisenhauer, C., "Compendium of Benchmark Neutron Fields For Reactor Dosimetry," NBSIR 85-3151, National Institute of Standards and Technology, Gaithersburg, MD Jan. 1986.

[11] Kocherov, N.P., et al., "International Reactor Dosimetry File(IRDF-90)," IAEA-NDS-141 Rev 0, International Atomic Energy Agency, August 1990, Brookhaven National Laboratory, Upton ,New York

[12] Kaul, D.C.\& Egbert, S.D., "Radiation Leakage from the Army Pulse Radiation Facility Fast Reactor," SAIC-89/1423, Science Applications International Corporation, San Diego, CA, May 1989. 